07

\title{
Генерация и релаксация напряжений в гетероструктуре (Al, Ga)N/6H-SiC при росте методом плазменно-активированной молекулярно-пучковой эпитаксии
}

\author{
(C) Д.В. Нечаев, А.А. Ситникова, П.Н. Брунков, С.В. Иванов, \\ В.Н. Жмерик
}

Физико-технический институт им. А.Ф. Иоффе РАН, Санкт-Петербург E-mail: nechayev@mail.ioffe.ru

Поступило в Редакцию 13 декабря 2016 г.

Исследованы in situ генерация и релаксация напряжений в гетероструктуре $\mathrm{Al}_{0.25} \mathrm{Ga}_{0.75} \mathrm{~N} / \mathrm{GaN} / \mathrm{AlN}$ общей толщиной более $3 \mu \mathrm{m}$ в процессе ее роста на подложке $6 H$-SiC методом низкотемпературной плазменно-активированной молекулярно-пучковой эпитаксии при температурах подложки $690-740^{\circ} \mathrm{C}$. При комнатной температуре слои $\mathrm{AlN}$ и $\mathrm{GaN}$ показали остаточные сжимающие напряжения величиной -2.3 и $-0.1 \mathrm{GPa}$ соответственно, что позволило исключить растрескивание структуры при ее постростовом остывании.

DOI: 10.21883/PJTF.2017.09.44578.16607

Для производства высокочастотных, мощных и радиационно-стойких транзисторов необходимы качественные гетероструктуры (ГС) в системе материалов (Al, Ga)N. Вследствие отсутствия коммерчески доступных гомоэпитаксиальных подложек рост транзисторных ГС методами газофазной и молекулярно-пучковой эпитаксии (МПЭ) проводится на гетероподложках $6 \mathrm{H}-\mathrm{SiC}, c-\mathrm{Al}_{2} \mathrm{O}_{3}, \mathrm{Si}(111)$ и др. Первые, наряду с высокой теплопроводностью (даже по сравнению с $4 \mathrm{H}$-SiC [1]), обладают наименьшим рассогласованием $\left(\varepsilon_{m}\right) \alpha$-постоянных решеток подложки $6 H$-SiC $\left(a_{s}\right)$ и буферного слоя $\mathrm{AIN}\left(a_{f}\right) \varepsilon_{m}=\left(a_{s}-a_{f}\right) / a_{s}=-1 \%$ [2]. Однако, несмотря на это относительно небольшое рассогласование, на первых этапах развития технологий при росте буферных слоев $\mathrm{AlN} / 6 H-\mathrm{SiC}$ наблюдались высокие плотности прорастающих дислокаций (ПД) (до $>10^{10} \mathrm{~cm}^{-2}$ ) [3]. Это связывалось с грубой морфологией 
поверхности подложек и низким структурным качеством начальных слоев ГС. Лишь по мере улучшения качества предэпитаксиальной химико-механической полировки подложек и оптимизации начальных условий роста концентрации ПД в активных областях ГС были снижены до значений $10^{8}-10^{9} \mathrm{~cm}^{-2}[4]$.

Другой существенной проблемой роста $(\mathrm{Al}, \mathrm{Ga}) \mathrm{N}$ ГС является образование трещин в буферных слоях $\mathrm{AlN} / 6 H$-SiC вследствие генерации в них растягивающих напряжений, обусловленных развитием в них внутренней зеренной структуры [3], несмотря на обратный знак кристаллографического рассогласования постоянных решетки в этой ГС. Кроме того, растягивающие напряжения всегда вводятся в транзисторные ГC с Ga-содержащими слоями $(\mathrm{Al}, \mathrm{Ga}) \mathrm{N}$ во время их постростового охлаждения из-за различия линейных коэффициентов теплового расширения $(\alpha)$ для этих слоев $\left(\alpha_{\mathrm{GaN}}=5.59 \cdot 10^{-6} \mathrm{~K}^{-1}[5]\right)$ и подложки $6 H-\mathrm{SiC}\left(\alpha_{\mathrm{AIN}}=\alpha_{\mathrm{SiC}}=4.2 \cdot 10^{-6} \mathrm{~K}^{-1}[6]\right)$.

Согласно оценкам, выполненным с помощью различных моделей, значение критической толщины для гетероструктуры $\mathrm{AlN} / 6 \mathrm{H}-\mathrm{SiC}$ coставляет от 3.5 до $20 \mathrm{~nm}$ [7]. Действительно, при росте зародышевых слоев AlN c помощью высокотемпературной газофазной эпитаксии (температура подложки $T_{S}>1000^{\circ} \mathrm{C}$ ) наблюдаются практически полная релаксация напряжений в самом начале роста [8] и даже переход к растягивающим напряжениям при дальнейшем росте [3]. При росте следующих буферных слоев $\mathrm{GaN}$ наблюдается сильная степень релаксации начальных сжимающих напряжений в ГC GaN/AlN. B результате все эти процессы вместе с генерацией постростовых растягивающих напряжений могут приводить к растрескиванию (cracking) приборных ГС [9].

Наилучшим решением этой проблемы является развитие методов псевдоморфного роста слоев в транзисторных ГС AlGaN/GaN/AlN/6H$\mathrm{SiC}$, что в принципе должно приводить к генерации во всех слоях этой ГС сжимающих напряжений, которые необходимы для компенсации растягивающих напряжений во время ее постростового остывания. При этом достижение более высокого уровня сжимающих напряжений в ГС позволит выращивать более толстые буферные слои $\mathrm{GaN}$, что, как правило, приводит к снижению плотности ПД в верхних (активных) областях ГС. Кроме того, поскольку основными механизмами релаксации напряжений являются введение различных дефектов и переход к трехмерному росту, то псевдоморфный рост должен приводить к снижению

Письма в ЖТФ, 2017, том 43, вып. 9 
дефектности ГС и двумерному росту. Для решения этой задачи привлекательным выглядит развитие низкотемпературных методов эпитаксии. Так, в [7] при использовании плазменно-активированной (ПА) МПЭ с относительно низкой температурой эпитаксиального роста $T_{S} \sim 650^{\circ} \mathrm{C}$ (вместо $T_{S}=1200-1300^{\circ} \mathrm{C}$, используемых в газофазных эпитаксиальных технологиях) наблюдался псевдоморфный рост слоев AlN на подложках $6 H$-SiC вплоть до толщин $>700 \mathrm{~nm}$, что было объяснено авторами активационным барьером образования дислокаций [10].

В данной статье исследуются генерация и релаксация напряжений в ГC $\mathrm{Al}_{0.25} \mathrm{Ga}_{0.75} \mathrm{~N} / \mathrm{GaN} / \mathrm{AlN}$ с суммарной толщиной более $3 \mu \mathrm{m}$ во время ее низкотемпературного роста $\left(T_{S}<740^{\circ} \mathrm{C}\right)$ методом ПА МПЭ на подложке $6 \mathrm{H}$-SiC. В результате демонстрируются остаточные сжимающие напряжения в этой ГС, что объясняется возможностями ПА МПЭ по реализации двумерного роста $\mathrm{AlGaN}$ в различных импульсных режимах в металлобогащенных условиях, способствующих кинетическому ограничению зарождения и распространения ПД.

ГС выращивалась на установке ПА МПЭ Compact 21T (Riber). Подложка $6 \mathrm{H}-\mathrm{SiC}$ с двухсторонней полировкой и нанесенным на обратную сторону слоем Ті толщиной $200 \mathrm{~nm}$ отжигалась при максимально возможной $T_{S}=740^{\circ} \mathrm{C}$ в течение $40 \mathrm{~min}$. Затем для ее дополнительной очистки проводились два цикла осаждения/испарения нескольких монослоев (ML) Ga при температуре $T_{S}=600 / 740^{\circ} \mathrm{C}$. Зародышевый слой AlN толщиной $65 \mathrm{~nm}$ был выращен в режиме эпитаксии с повышенной миграцией (ЭПМ) [11], в котором использовалась попеременная подача ростовых потоков $\mathrm{Al}$ и $\mathrm{N}^{*}$ с номинальными значениями соответствующих толщин $F_{\mathrm{Al}}=F_{\mathrm{N}^{*}}=6 \mathrm{ML}$ в каждом цикле. Дальнейший рост слоя AlN толщиной $455 \mathrm{~nm}$ проводился посредством металлмодулированной эпитаксии (ММЭ) [12] в металлобогащенных условиях $\left(F_{\mathrm{Al}} / F_{\mathrm{N}^{*}}>1.2\right)$ при $T_{S}=740^{\circ} \mathrm{C}$. Во время роста слоя $\mathrm{AlN}$ на его поверхность в качестве сурфактанта подавался поток $\mathrm{Ga}$ $F_{\mathrm{Ga}}=0.3 \mathrm{ML} / \mathrm{s}$. Слой $\mathrm{GaN}$ толщиной $2.8 \mu \mathrm{m}$ выращивался с помощью ММЭ при $F_{\mathrm{Ga}} / F_{\mathrm{N}^{*}}>2.3$ и $T_{S}=690^{\circ} \mathrm{C}$. Верхний слой $\mathrm{Al}_{0.25} \mathrm{Ga}_{0.75} \mathrm{~N}$ толщиной $18 \mathrm{~nm}$ выращивался в слегка металлобогащенных условиях.

Для in situ контроля морфологии поверхности и скорости роста слоев использовались дифракция отраженных быстрых электронов (ДОБЭ) и лазерная рефлектометрия. С помощью многолучевого оптического измерителя напряжений (МОИН) непрерывно измерялась кривизна подложки $(\kappa)$, что позволило оценивать произведе-

Письма в ЖТФ, 2017, том 43, вып. 9 


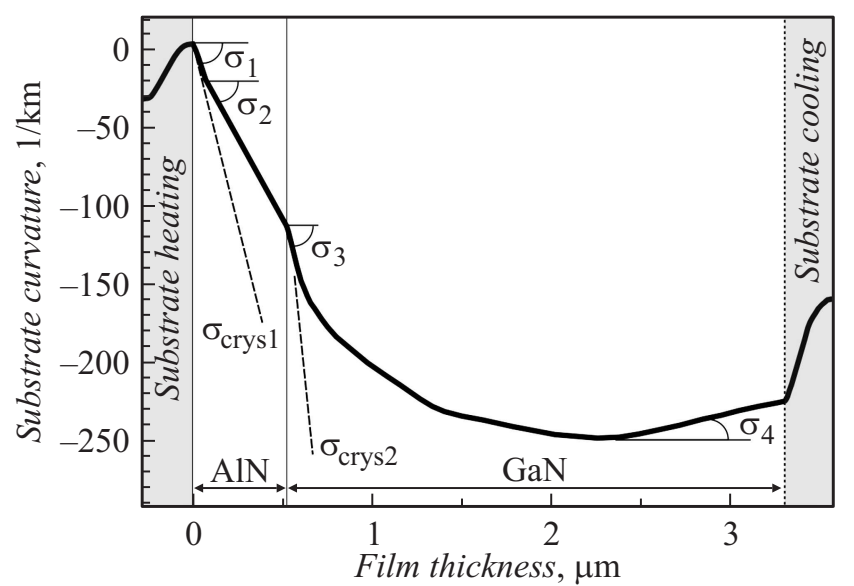

Рис. 1. Данные МОИН во время роста слоев $\mathrm{AlN}$ и $\mathrm{GaN}$ с инкрементальными напряжениями $\sigma_{1}=-3 \mathrm{GPa}, \sigma_{2}=-2.2 \mathrm{GPa}, \sigma_{3}=-6.3 \mathrm{GPa}, \sigma_{4}=+0.4 \mathrm{GPa}$. Штриховыми линиями обозначен псевдоморфный рост слоев $\mathrm{AlN}$ и $\mathrm{GaN}$ с напряжениями $\sigma_{\text {crys } 1}=-4.7 \mathrm{GPa}$ и $\sigma_{\text {crys } 2}=-11.2 \mathrm{GPa}$ соответственно.

ние средней величины напряжений $\left(\left\langle\sigma_{f}\right\rangle\right)$ на толщину слоя $\left(h_{f}\right)$ через соотношение Стоуни (Stoney) $\left\langle\sigma_{f}\right\rangle h_{f}=M_{s} h_{s}^{2} \kappa / 6$ [13], где $h_{s}-$ толщина подложки, а $M_{s}=602 \mathrm{GPa}$ - биаксиальный модуль упругости подложки $6 H-\mathrm{SiC}$ [14]. Для расчетов инкрементальных напряжений $\left(\sigma_{f}\left(h_{f}\right)\right)$ использовалось дифференциальное соотношение Стоуни $\sigma_{f}\left(h_{f}\right)=M_{s} h_{s}^{2} d \kappa\left(h_{f}\right) / 6 d\left(h_{f}\right)$. Кристаллографические напряжения несоответствия $\left(\sigma_{\text {crys }}\right)$, соответствующие псевдоморфному росту, рассчитывались по формуле $\sigma_{\text {crys }}=M_{\mathrm{A}_{3} \mathrm{~N}} \varepsilon_{m}$, где $M_{\mathrm{AlN}}=470 \mathrm{GPa}$ и $M_{\mathrm{GaN}}=449 \mathrm{GPa}$ - биаксиальные модули упругости слоев $\mathrm{A}_{3} \mathrm{~N}$ согласно работе Wright [15]. Степень релаксации этих напряжений $(\gamma)$ вычислялась как $\gamma=1-\sigma_{f} / \sigma_{\text {crys. }}$. Морфология поверхности слоев и их структура исследовались на оборудовании федерального ЦКП „Материаловедение и диагностика в передовых технологиях“ с помощью оптического микроскопа, атомно-силовой микроскопии (ACM) и просвечивающей электронной микроскопии (ПЭМ, JЕM 2100-F).

Рис. 1 демонстрирует изменение кривизны подложки на стадиях нагрева, роста $\Gamma \mathrm{C} \mathrm{Al}_{0.25} \mathrm{Ga}_{0.75} \mathrm{~N} / \mathrm{GaN} / \mathrm{AlN}$ и ее охлаждения. Положитель- 
$a$

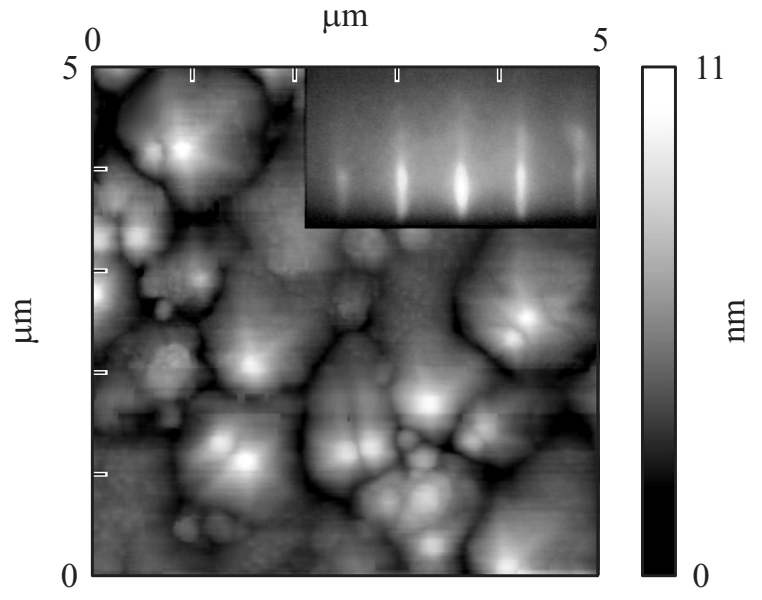

$b$

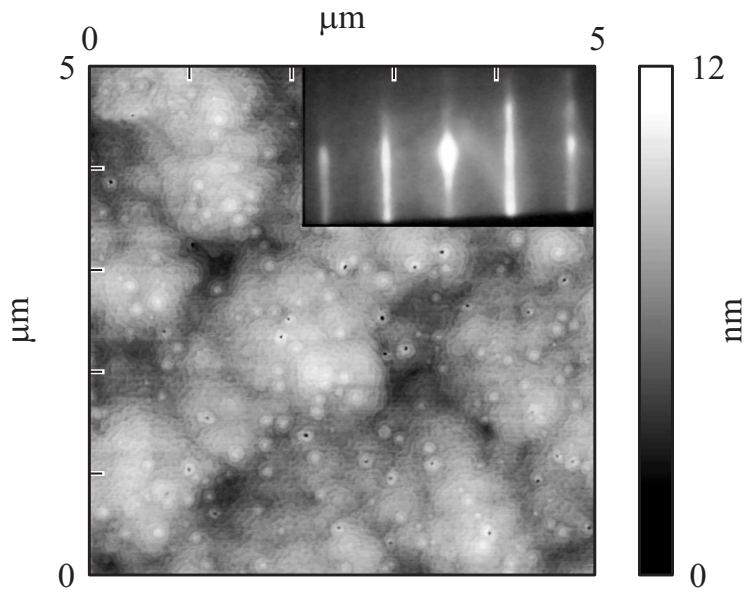

Рис. 2. АСМ-изображения поверхности слоев $\mathrm{AlN}(a)$ и $\mathrm{GaN}(b)$ с шероховатостью $\mathrm{RMS}=1.5$ и $1 \mathrm{~nm}$ на площади $5 \times 5 \mu \mathrm{m}$ соответственно. Слои были выращены в импульсных металлобогащенных условиях роста при $T_{s}=740$ и $690^{\circ} \mathrm{C}$ соответственно.

ное изменение кривизны $\Delta \kappa=35 \mathrm{~km}^{-1}$ во время предростового нагрева подложки объясняется генерацией термических напряжений в слое Ті.

Письма в ЖТФ, 2017, том 43, вып. 9 
Наблюдающееся при росте зародышевого слоя AlN линейное отрицательное изменение кривизны подложки соответствует сжимающим напряжениям $\left\langle\sigma_{f}\right\rangle=\sigma_{1}=-3 \mathrm{GPa}$, что при значении $\sigma_{\text {cryst }}=-4.7 \mathrm{GPa}$ соответствует степени релаксации $\gamma=36 \%$. При росте буферного слоя AIN с использованием ММЭ наблюдаются меньшие напряжения $\left\langle\sigma_{f}=\sigma_{2}=-2.2 \mathrm{GPa}\right.$, что связано с развитием процесса коалесценции зерен в конце роста зародышевого и начале роста буферного слоев [16]. $\mathrm{B}$ итоге средние напряжения в слое $\mathrm{AlN}$ составили $\left\langle\sigma_{f}\right\rangle=-2.3 \mathrm{GPa}$.

Напряжения $\sigma_{3}=-6.3 \mathrm{GPa}$ наблюдаются и в начальный момент роста буферного слоя $\mathrm{GaN}$ на слое $\mathrm{AlN}$, что при значении $\sigma_{\text {crys } 2}=-11.2 \mathrm{GPa}$ соответствует степени релаксации напряжений $\gamma=44 \%$. Однако при дальнейшем росте этого слоя наблюдается постепенная релаксация напряжений, а при достижении им толщины $\sim 1.8 \mu \mathrm{m}$ происходит переход к генерации растягивающих напряжений с постоянной величиной $\sigma_{4}=+0.4 \mathrm{GPa}$, которые сохраняются вплоть до конца роста $\mathrm{GaN}\left(\left\langle\sigma_{f}\right\rangle=-0.49 \mathrm{GPa}\right)$. Такое поведение напряжений в слое $\mathrm{GaN}$, скорее всего, вызвано наклоном прорастающих дислокаций, что, согласно модели Romanov \& Speck, должно приводить к инверсии знака напряжений в ГC GaN/AlN [17]. Однако эта модель не может объяснить наблюдающуюся генерацию постоянных растягивающих напряжений в слое $\mathrm{GaN}$, и эта проблема требует дополнительных исследований.

При остывании ГС наблюдается положительное изменение кривизны подложки $\Delta \kappa=65 \mathrm{~km}^{-1}$, которое соответствует термическим $\left(\sigma_{t h}\right)$ растягивающим напряжениям $\sigma_{t h}=+0.39 \mathrm{GPa}$ (с учетом влияния слоя Ti, который изгибал подложку дополнительно на $\Delta \kappa=-35 \mathrm{~km}^{-1}$ ), что достаточно близко к их расчетной величине $\sigma_{t h}=+0.42 \mathrm{GPa}$. B результате при комнатной температуре наблюдались остаточные средние сжимающие напряжения в слоях $\mathrm{AlN}$ и $\mathrm{GaN}$ величиной $\left\langle\sigma_{\text {res }}\right\rangle=-2.3$ и $-0.1 \mathrm{GPa}$ соответственно, вычисленные по формуле $\left\langle\sigma_{\text {res }}\right\rangle=\left\langle\sigma_{f}\right\rangle+\sigma_{t h}$. Отсутствие трещин в образце было подтверждено оптической микроскопией.

Сжимающие напряжения в слое AIN и значительной части вышележащего слоя $\mathrm{GaN}$ можно объяснить следующими причинами. Boпервых, это вызвано высоким качеством подложки $6 \mathrm{H}-\mathrm{SiC}$, обладающей атомарно-гладкой морфологией поверхности с шероховатостью $\mathrm{RMS}=0.14 \mathrm{~nm}$ на площади $2 \times 5 \mu \mathrm{m}$. Во-вторых, металлобогащенные условия роста слоев $\mathrm{AlN}$ и $\mathrm{GaN}$ импульсными методами ЭПМ и ММЭ

Письма в ЖТФ, 2017, том 43, вып. 9 

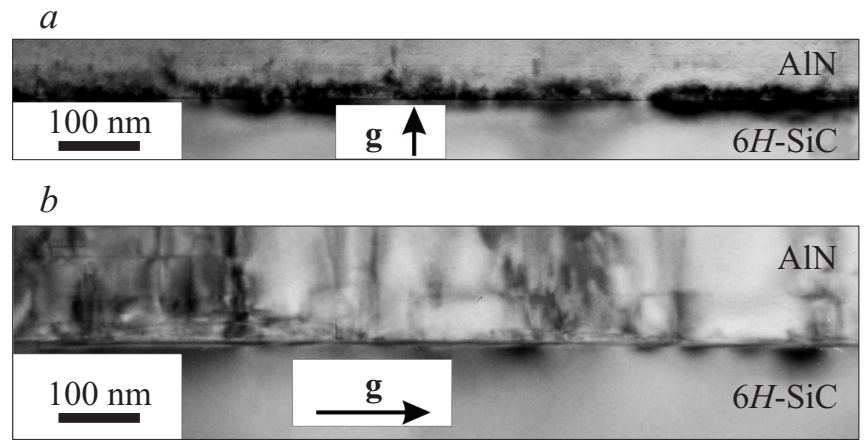

Рис. 3. Поперечные ПЭМ-изображения гетерограницы $\mathrm{AlN} / 6 H-\mathrm{SiC}$, полученные при различных ориентациях вектора дифракции $\mathbf{g}=(0002)(a)$ и $\mathbf{g}=(01 \overline{1} 0)(b)$.

способствовали поддержанию двумерного механизма эпитаксиального роста, что подтверждалось линейчатой формой рефлексов картин ДОБЭ. Из АСМ-изображений слоев $\mathrm{AlN}$ и $\mathrm{GaN}$ на рис. 2 следует, что их рост протекал согласно спиральному ступенчато-слоевому механизму, который позволил достичь значений шероховатости на площади $5 \times 5 \mu \mathrm{m}$ RMS $\sim 1.5$ и $1 \mathrm{~nm}$ соответственно. И наконец, росту со сжимающими напряжениями на начальных этапах, по-видимому, способствовало существование при низкотемпературном росте $\left(T_{s}<740^{\circ} \mathrm{C}\right)$ кинетических ограничений на зарождение различных дефектов на гетерогранице $\mathrm{AlN} / 6 \mathrm{H}-\mathrm{SiC}$, включая различные типы дислокаций, дефекты упаковки и др. На это указывают ПЭМ-изображения гетерограницы (рис. 3) с относительно невысокими концентрациями ПД по сравнению с ростом аналогичных слоев $\mathrm{AlN}$ на $c-\mathrm{Al}_{2} \mathrm{O}_{3}[11]$.

Таким образом, с помощью МОИН исследованы процессы генерации и релаксации упругих напряжений в ГC $\mathrm{AlGaN} / \mathrm{GaN} / \mathrm{AlN} / 6 H$ $\mathrm{SiC}$ при ее росте методом низкотемпературной $\left(T_{S}=740^{\circ} \mathrm{C}\right)$ ПА МПЭ. В частности, продемонстрированы высокие остаточные сжимающие напряжения в толстом слое $\mathrm{AlN}\left\langle\sigma_{\text {res }}\right\rangle=-2.3 \mathrm{GPa}$ и сложные процессы генерации и релаксации напряжений в слое $\mathrm{GaN}$ толщиной $2.8 \mu \mathrm{m}$, обусловившие в итоге остаточные сжимающие напряжения $\left\langle\sigma_{\text {res }}\right\rangle=-0.1 \mathrm{GPa}$. Показаны преимущества низкотемпературной ПА МПЭ по сравнению с более высокотемпературными методами эпи-

Письма в ЖТФ, 2017, том 43, вып. 9 
таксии для роста сжатых слоев $\mathrm{AlN}$ и GaN с суммарной толщиной несколько микрон на подложках $6 \mathrm{H}-\mathrm{SiC}$, что позволяет решить проблему растрескивания приборных ГС при их постростовом остывании.

Работа выполнена при поддержке РФФИ (грант № 16-32-00844 мол_а).

\section{Список литературы}

[1] Kukushkin S.A., Osipov A.V., Bessolov V.N. et al. // Rev. Adv. Mater. Sci. 2008. V. 17. P. 1.

[2] Liu L., Edgar J.H. // Mater. Sci. Eng. R. 2002. V. 37. P. 61.

[3] Taniyasu Y., Kasu M., Makimoto T. // J. Cryst. Growth. 2007. V. 298. P. 310.

[4] Okumura H., Kimoto T., Suda J. // Appl. Phys. Exp. 2011. V. 4. P. 025502.

[5] Maruska H.P., Tietjen J.J. // Appl. Phys. Lett. 1969. V. 15. P. 327.

[6] Yim W.M., Paff R.J. // J. Appl. Phys. 1974. V. 45. P. 1456.

[7] Okumura H., Kimoto T., Suda J. // Appl. Phys. Exp. 2012. V. 5. P. 105502.

[8] Cho E., Mogilatenko A., Brunner F. et al. // J. Cryst. Growth. 2013. V. 371. P. 45.

[9] Guojian D., Liwei G., Zhigang X. et al. // J. Semicond. 2010. V. 31. P. 033003.

[10] Lacey G., Whitehouse C.R., Parbrook P.J. et al. // Appl. Surf. Sci. 1998. V. 123/124. P. 718.

[11] Nechaev D.V., Aseev P.A., Jmerik V.N. et al. // J. Cryst. Growth. 2013. V. 378. P. 319.

[12] Jmerik V.N., Mizerov A.M., Nechaev D.V. et al. // J. Cryst. Growth. 2012. V. 354. P. 188.

[13] Hearne S., Chason E., Han J. et al. // Appl. Phys. Lett. 1999. V. 74. P. 356.

[14] Kamitani K., Grimsditch M., Nipko J.C. et al. // J. Appl. Phys. 1997. V. 82. P. 3152.

[15] Wright A.F. // J. Appl. Phys. 1997. V. 82. P. 2833.

[16] Nix W.D., Clemens B.M. // J. Mater. Res. 1999. V. 14. P. 3467.

[17] Cantu P., Wu F., Waltereit P. et al. // J. Appl. Phys. 2005. V. 97. P. 103534. 